\title{
The Hsp70-like StkA functions between T4P and Dif signaling proteins as a negative regulator of exopolysaccharide in Myxococcus xanthus
}

Pamela L. Moak, Wesley P. Black, Regina A. Wallace, Zhuo Li, Zhaomin Yang

Myxococcus xanthus displays a form of surface motility known as social (S) gliding. It is mediated by the type IV pilus (T4P) and requires the exopolysaccharide (EPS) to function. It is clear that T4P retraction powers S motility. EPS on a neighboring cell or deposited on a gliding surface is proposed to anchor the distal end of a pilus and trigger T4P retraction at its proximal end. Inversely, T4P have been shown to regulate EPS production upstream of the Dif signaling pathway. Here we describe the isolation of two Tn insertions at the stk locus which had been known to play roles in cellular cohesion and formation of cell groups. An insertion in stKA (MXAN_3474) was identified based on its ability to restore EPS to a pilA deletion mutant. stkA encodes a DnaK or Hsp70 homolog and it is upstream of the stkB (MXAN_3475) and stkC (MXAN_3476) genes. A stkB insertion was identified in a separate genetic screen because it eliminated EPS production of an EPS ${ }^{+}$parental strain. Our results with in-frame deletions of these three stk genes indicated that the stkA mutant produced increased level of EPS while $s t k B$ and stkC mutants produced less EPS relative to the wild type. $S$ motility and developmental aggregation were affected by deletions of StkA and $s t k B$ but only minimally by the deletion of $s t k C$. Genetic epistasis indicated that StkA functions downstream of T4P but upstream of the Dif proteins as a negative regulator of EPS production in $M$. xanthus. 
The Hsp70-like StkA functions between T4P and Dif signaling proteins as a negative regulator of exopolysaccharide in Myxococcus xanthus

4

7 Department of Biological Sciences, Virginia Polytechnic Institute and State University, Blacksburg,

8 Virginia 24061

9

$10 *$ Corresponding author: Zhaomin Yang

11 Phone: 540-231-1350; email: zmyang@,vt.edu

12

13 Short title: Hsp70 in M. xanthus EPS regulation

14

15 Keywords: Myxococcus xanthus; type IV pilus (T4P); Exopolysaccharide (EPS); social motility; Dif 16 pathway; StkA/DnaK/Hsp70 
18 ABSTRACT Myxococcus xanthus displays a form of surface motility known as social (S) gliding. It is

20 mediated by the type IV pilus (T4P) and requires the exopolysaccharide (EPS) to function. It is clear

21 that T4P retraction powers S motility. EPS on a neighboring cell or deposited on a gliding surface is

22 proposed to anchor the distal end of a pilus and trigger T4P retraction at its proximal end. Inversely,

23 T4P have been shown to regulate EPS production upstream of the Dif signaling pathway. Here we

24 describe the isolation of two Tn insertions at the stk locus which had been known to play roles in

25 cellular cohesion and formation of cell groups. An insertion in stkA (MXAN_3474) was identified

26 based on its ability to restore EPS to a pilA deletion mutant. stkA encodes a DnaK or Hsp70 homolog

27 and it is upstream of the stkB (MXAN_3475) and stkC (MXAN_3476) genes. A stkB insertion was

28 identified in a separate genetic screen because it eliminated EPS production of an EPS ${ }^{+}$parental strain.

29 Our results with in-frame deletions of these three stk genes indicated that the $s t k A$ mutant produced

30 increased level of EPS while $s t k B$ and $s t k C$ mutants produced less EPS relative to the wild type. S

31 motility and developmental aggregation were affected by deletions of $s t k A$ and $s t k B$ but only minimally

32 by the deletion of $s t k C$. Genetic epistasis indicated that StkA functions downstream of T4P but

33 upstream of the Dif proteins as a negative regulator of EPS production in M. xanthus. 


\section{INTRODUCTION}

36 Myxococcus xanthus, a gram negative bacterium, exhibits complex social interactions during its life

37 cycles (Yang \& Higgs 2014). When nutrients are plentiful, M. xanthus cells grow, divide and move

38 over solid surfaces as social swarms in a vegetative growth cycle. Upon nutrient limitation, M. xanthus

39 initiates a developmental cycle wherein cells aggregate on solid surfaces by their gliding motility.

40 When these aggregates mature into multicellular fruiting bodies, rod-shaped vegetative cells within

41 morph into spherical myxospores. These metabolically dormant myxospores can endure adverse

42 environmental elements such as heat, desiccation and UV radiation. When conditions become

43 conducive for growth, myxospores germinate to reenter the vegetative cycle. Both developmental

44 fruiting and vegetative swarming are multicellular behaviors which make M. xanthus a good model to 45 study social or cell-cell interactions.

46 M. xanthus uses two genetically and morphologically distinct surface motility systems to facilitate its

47 vegetative swarming and developmental aggregation (Mauriello et al. 2010). The adventurous (A)

48 gliding system enables cells to move even when they are well isolated from one another. Social (S)

49 gliding, on the other hand, only functions when cells are in close proximity or in groups. The bacterial

50 type IV pilus (T4P) is known as the engine whose retraction powers $M$. xanthus $\mathrm{S}$ motility and

51 bacterial twitching (Mauriello et al. 2010). Besides T4P, M. xanthus $\mathrm{S}$ motility requires the

52 extracellular or exo-polysaccharide (EPS) to function (Yang et al. 2014). Available evidence supports

53 a model wherein EPS on a neighboring cell or a surface triggers the T4P of M. xanthus to retract to

54 actualize S motility (Li et al. 2003). This model explains why the function of S motility requires both

55 T4P and EPS as well as cell proximity on most surfaces examined. 
The T4P or Pil proteins as well as the Dif pathway play key roles in EPS regulation in $M$.

57 xanthus (Black et al. 2006; Yang et al. 2014). pilA and other T4P- pil mutants have been found to be

58 EPS- A pilT mutant, which is $\mathrm{T}^{-} \mathrm{P}^{+}$with non-retractable $\mathrm{T} 4 \mathrm{P}$, is EPS ${ }^{+}$. There is therefore a positive

59 correlation between the presence of T4P and EPS. Genes at the dif locus encode products related to

60 bacterial chemotaxis proteins (Yang et al. 1998b). DifA is homologous to the chemoreceptor MCP,

61 DifC to the coupling protein $\mathrm{CheW}$, and DifE to the histidine kinase CheA. Two additional proteins

62 DifD and DifG are similar to the response regulator CheY and the phosphatase CheC, respectively.

63 Null mutations in $\operatorname{difA}$, $\operatorname{difC}$ and $\operatorname{difE}$ led to EPS- and those in $\operatorname{difD}$ and $\operatorname{dif} G$ to EPS overproduction

64 (Black \& Yang 2004; Yang et al. 2000). Evidence indicated that DifE is a protein kinase and that it

65 forms a ternary signaling complex with DifA and DifC (Black et al. 2010; Yang \& Li 2005). DifD and

66 DifG influence the signaling strength of this DifACE complex by diverting phosphates from the kinase.

67 Genetic studies showed that the T4P functions upstream of the Dif signaling proteins in EPS regulation

68 (Black et al. 2006). The current model proposes that T4P function as physical sensors of other cells

69 nearby. This sensory information is then relayed to the Dif pathway downstream to promote EPS

70 production.

Of additional relevance to this work are the genes at the stk and the che 7 loci. A frameshift

72 mutation in cheW7 (cheW7-1) in the che7 gene cluster (Zusman et al. 2007) restored EPS production to

73 a difA deletion ( $\triangle$ dif $A$ ) mutant (Black et al. 2009). That is, a $\triangle d i f A$ single mutant is EPS- but a $\triangle$ dif $A$

74 cheW7-1 double mutant is EPS ${ }^{+}$. The Che7 chemosensory system likely plays an accessory role in EPS

75 regulation in $M$. xanthus because a cheW7 null mutation by itself does not impact EPS production in an

76 otherwise wild-type (WT) background (Black et al. 2009). The stk locus had been identified previously

77 because its mutations enhanced cellular cohesion in liquid culture and increased group formation at

78 colony edges (Dana \& Shimkets 1993b). Here we describe the isolation of two transposon insertions at 
79 the stk locus and the genetic characterization of stkA (MXAN_3474), stkB (MXAN_3475) and stkC

80 (MXAN_3476). A stkA insertion was found to suppress the EPS defect of a $\triangle p i l A$ mutant whereas a

$81 s t k B$ insertion was found to eliminate EPS production of a $\triangle$ difA cheW7-1 strain. StkA is homologous

82 to DnaK and HSP70 as described previously (Weimer et al. 1998). StkB shares similarity with the

83 sterol carrier protein 2 (SCP2) or nonspecific lipid-transfer protein (NSLTP) (Lev 2010; Schroeder et

84 al. 2007). StkC is a small protein with limited homology to PhaE (Goldman et al. 2006), an enzyme

85 involved in polyhydroxyalkanoate synthesis (Han et al. 2010). In-frame deletions were constructed for

86 all three $s t k$ genes and their mutants were studied phenotypically. $s t k B$ and $s t k C$ deletions led to

87 intermediate phenotypes in EPS production, motility and fruiting body development. Both stkA

88 insertion and deletion restored EPS production to a $\triangle p i l A$ mutant, but they failed to do so to a $\triangle$ difA

89 strain. These results support a model wherein StkA functions downstream of T4P but upstream of the

90 Dif pathway in the regulation of M. xanthus EPS production as a negative regulator. StkB and StkC are

91 required for EPS production at the wild-type level and the absence of either reduced but did not

92 eliminate EPS production. 


\section{MATERIALS \& METHODS}

\section{Bacterial strains and growth conditions}

96 Escherichia coli DH5 $\alpha$ was used for plasmid constructions while DH5 $\alpha \lambda$ pir was used to clone

97 transposon insertions from M. xanthus mutants. They were grown and maintained on Luria Bertani

98 (LB) agar plates or in LB liquid medium (Sambrook \& Russell 2001). M. xanthus strains used in this

99 study are listed in Table 1 and they were grown and maintained on Casitone yeast extract (CYE) agar

100 plates or in its liquid form (Campos \& Zusman 1975). Clone-fruiting (CF) agar plates were used to

101 examine fruiting body development (Hagen et al. 1978). Plates for general use contained $1.5 \%$ agar.

102 Soft agar plates, which were used to examine S motility, contained 0.4\% agar (Shi \& Zusman 1993).

103 Whenever necessary, kanamycin and oxytetracycline were supplemented to media at $100 \mu \mathrm{g} / \mathrm{ml}$ and 15

$104 \mu \mathrm{g} / \mathrm{ml}$, respectively (Bellenger et al. 2002; Black \& Yang 2004).

105 Transposon mutagenesis and identification of transposon insertions

106 Transposon mutagenesis was performed using the mariner-based magellan4 (Rubin et al. 1999) as

107 previously described (Black et al. 2009). pMycoMar (containing magellan4) (Table 1) (Rubin et al.

108 1999) was electroporated into YZ101 (AdifA cheW7-1) or DK10407 (ApilA). Cells were allowed to

109 recover for 4 hours and plated on CYE plates with Congo Red at $30 \mu \mathrm{g} / \mathrm{ml}$. About 20,000 colonies

110 were visually screened for EPS phenotypes after 5-7 days of incubation at $32^{\circ} \mathrm{C}$ (Black et al. 2009).

111 The site of a Tn insertion in a mutant of interest was identified by cloning and DNA sequencing as has

112 been described (Black et al. 2009). Briefly, genomic DNA from a mutant was digested with SacII

113 (New England Biolabs) and allowed to self ligate. The ligation was transformed into DH5 $\alpha$ pir. Two 
114 primers, MarR1 and/or MarL1 (Youderian et al. 2003) were used to sequence the plasmids recovered

115 from the transformant.

\section{Construction of plasmids and strains}

117 Plasmids used in this study are listed in Table 1. In-frame deletion alleles of stk genes were 118 constructed using a two-step overlap PCR procedure (Sambrook \& Russell 2001). PCR products with

119 the in-frame deletion alleles of $s t k A$ and $s t k C$ were blunt-end ligated into SmaI-digested pBJ113

120 (Julien et al. 2000) create pLZ407 and pAM108, respectively. The PCR product with the stkB in-frame

121 deletion was similarly ligated into SmaI-restricted pBJ114 (Julien et al. 2000) to create pLZ429. The

122 mutant alleles in pLZ407, pLZ429 and pAM108 deleted codons 5 to 535 for StkA, 5 to 108 for StkB

123 and 5 to 85 for StkC, respectively.

124 A two-step procedure (Ueki et al. 1996) was performed to construct deletions of chromosomal stk

125 genes. The three plasmids above were used to delete $s t k A$, stkB and $s t k C$ from the WT strain (DK1622)

126 to construct YZ812 ( $\Delta$ stkA), YZ813 ( $\Delta$ stkB) and YZ910 ( $\Delta$ stkC), respectively. In addition, pLZ407

127 was used to delete stkA from YZ690 ( $\triangle$ pilA) and YZ601 ( $\Delta$ difA) to create strains YZ901 ( pila $\Delta$ stkA)

128 and YZ932 ( $\triangle$ difA $\Delta$ stkA).

\section{Examination of EPS Production}

130 EPS production was examined by two different assays, one qualitative and one quantitative. The

131 qualitative assay utilized plates with $50 \mu \mathrm{g} / \mathrm{ml}$ calcofluor white $(\mathrm{CW})$, a fluorescent dye that binds to

132 EPS (Black \& Yang 2004; Dana \& Shimkets 1993a). Cells from overnight cultures were pelleted and

133 resuspended in MOPS (morpholinepropanesulfonic acid) buffer (10 mM MOPS [pH 7.6], $2 \mathrm{mM}$

$134 \mathrm{MgSO}_{4}$ ) at approximately $5 \times 10^{9}$ cells $/ \mathrm{ml} .5 \mu \mathrm{l}$ of the suspension were spotted onto CYE plates with 
$135 \mathrm{CW}$ and incubated at $32^{\circ} \mathrm{C}$ for 6 days. Fluorescence under long-wavelength $(\sim 365 \mathrm{~nm}) \mathrm{UV}$ illumination

136 was directly photographed with a digital camera (Black et al. 2009). The binding of trypan blue was

137 used to quantify EPS in a liquid assay (Black \& Yang 2004). Cultures grown overnight in CYE to

$138 \sim 3.5 \times 10^{8}$ cells $/ \mathrm{ml}$ were harvested, washed and re-suspended to approximately $2.8 \times 10^{8}$ cells $/ \mathrm{ml} \mathrm{in}$

139 MOPS buffer with $5 \mu \mathrm{g} / \mathrm{ml}$ trypan blue. The control samples contained trypan blue in MOPS buffer

140 without cells. The samples were vortexed and incubated with shaking at $300 \mathrm{rpm}$ at $25^{\circ} \mathrm{C}$ for 30

141 minutes. The absorbance of the supernatants after centrifugation was measured at $585 \mathrm{~nm}$. EPS

142 production of all strains was normalized to that of the WT strain which was arbitrarily set as 1 (Dana \&

143 Shimkets 1993a). Quantitative experiments with trypan blue were repeated at least three times with

144 each sample analyzed in triplicates and a representative data set is shown in the paper.

\section{Examination of motility and fruiting body development}

146 Motility was examined by placing $5 \mu \mathrm{l}$ of the cell suspension at $5 \times 10^{9}$ cells $/ \mathrm{ml}$ onto the center of a

147 standard (1.5\% agar) or soft ( $0.4 \%$ agar) CYE plate. The standard agar plates were examined after 2

148 days and the soft agar plates after 5 days of incubation at $32^{\circ} \mathrm{C}$ (Black \& Yang 2004; Shi \& Zusman

149 1993). For the examination of fruiting body formation, overnight cultures were harvested and

150 resuspended in MOPS buffer at $5 \times 10^{9}$ cells $/ \mathrm{ml} .5 \mu$ of the suspension were spotted onto CF agar plates

151 and development was observed after 5 days of incubation at $32^{\circ} \mathrm{C}$. 


\section{RESULTS}

\section{Isolation of two $M$. xanthus transposon mutants with altered EPS production}

154 To identify genes involved in the regulation and/or production of EPS, two genetic screens were

155 carried out to search for mutants with altered EPS levels. In the first screen, a pilA deletion ( $\triangle$ pilA)

156 strain (DK10407), which is T4P- and EPS- (Black et al. 2006), was mutagenized by a transposon (Tn)

157 and mutants were allowed to form colonies on agar plates with the dye Congo red (CR). M. xanthus

$158 \mathrm{EPS}^{+}$colonies appear red and EPS- ones are yellowish orange on these plates (See Materials and

159 Methods). Among approximately 20,000 colonies screened, BY801 and BY802 were found to form red

160 colonies, indicating that they contained suppressors of the pilA deletion. BY801 is discussed here and

161 the work on BY802 has been published elsewhere (Wallace et al. 2014).

The suppressor phenotype of BY801 and its link to the Tn were confirmed by an alternative

163 EPS assay and genetic linkage analysis, respectively. As shown in Fig. 1A, BY801 was verified to be

$164 \mathrm{EPS}^{+}$as indicated by the fluorescence on a plate containing the dye Calcofluor white (CW). The Tn

165 insertion in BY801 was transferred to the parental ApilA mutant by genomic DNA transformation

166 (Vlamakis et al. 2004). Sixteen of the resulting transformants were examined and all were found to be

$167 \mathrm{EPS}^{+}$. This established that a single Tn insertion locus in BY801 was responsible for $\triangle$ pilA suppression

168 instead of any additional mutations elsewhere. When the Tn insertion was transferred to the WT

169 background, the resulting strain BY1801 showed enhanced EPS production as indicated by increased

$170 \mathrm{CW}$ binding in comparison with the wild type (WT) (Fig. 1A). These observations demonstrate that the

171 Tn insertion in BY801 altered the function of a gene or genes important for M. xanthus EPS production

172 and/or regulation. It should be noted that despite its ability to produce EPS, the colonies of BY801

173 differ from those of the WT (Fig. 1A) because the latter is $\mathrm{S}^{+}$while BY801 is $\mathrm{S}^{-}$without pilA. 
In the second genetic screen, the $\mathrm{EPS}^{+}$mutant YZ101 (cheW7-1 $\Delta$ difA) (Black et al. 2009) was

175 used for Tn mutagenesis to identify additional genes critical for M. xanthus EPS production. About 70

$176 \mathrm{EPS}^{-}$mutants were identified from approximately 20,000 colonies on CR plates. Some of these mutants

177 were reported previously (Black et al. 2009; Lu et al. 2005). BY129, which showed no obvious CR

178 binding in the initial screen, is described here. Examination on plates with CW confirmed that BY129

179 has negligible EPS levels in comparison with its parent and the WT (Fig 1B). When the insertion was

180 re-introduced into YZ101 by genomic DNA transformation, the resulting transformants displayed the

181 same EPS- phenotype as BY129 (not shown). When the Tn insertion was introduced into the WT

182 strain, the resulting mutant BY1129 did not bind CW in plate assays (Fig. 1B). The gene(s) mutated by

183 the Tn insertion in BY129 must play a role in EPS production and/or reguation in M. xanthus.

184 Transposons in BY801 and BY129 inserted in two adjacent genes at the stk locus

185 The Tn insertions in BY801 and BY129 were identified as previously described (Black et al. 2009). In

186 BY801, the insertion occured in MXAN_3474, a gene known as stk because of the sticky phenotype of

187 its mutant (Dana \& Shimkets 1993b; Kim et al. 1999; Weimer et al. 1998) (Fig. 2). This gene will be

188 designated as $s t k A$ and the insertion mutation here as $s t k A 2: \because$ Tn hereafter (Table 1 ). stkA encodes a

189 DnaK homologue of 540 amino acids (AAs) (Goldman et al. 2006) that was not found to be induced by

190 heat shock (Otani et al. 2001). The stkA2::Tn insertion occurred in the 440th codon of stkA after a TA

191 dinucleotide. In BY129, the Tn inserted into MXAN_3475, an open reading frame (ORF) of 141

192 codons 8 base pairs (bps) downstream of $s t k A$. This ORF will be designated as StkB and the mutation

193 as $s t k B 1:: T n$ hereafter. StkB belongs to the superfamily of the sterol carrier protein 2 (SCP2) or

194 nonspecific lipid-transfer protein (NSLTP) (Lev 2010; Schroeder et al. 2007). Some members of this

195 protein superfamily function in cholesterol trafficking and lipid metabolism as well as cell signaling in 
196 a variety of organisms (Lev 2010; Schroeder et al. 2007). The stkB1::Tn insertion occurred after a TA

197 dinucleotide in the 122nd codon. 12 bps downstream of stkB is MXAN_3476 (Goldman et al. 2006) or

$198 s t k C$. It encodes a protein of 89 AAs with limited homology to PhaE (Goldman et al. 2006), a

199 polyhydroxyalkanoate synthetic enzyme (Han et al. 2010). The isolation of $\mathrm{Tn}$ insertions at the stk

200 locus from independent genetic screens here and elsewhere (Dana \& Shimkets 1993b) indicates that

201 the stk genes are critical players in EPS production in M. xanthus.

$202 \Delta s t k A$ produces more EPS while $\Delta s t k B$ and $\Delta s t k C$ produce less

203 The transposons in BY801 and BY129 inserted at the $3^{\prime}$ ends of stkA and stkB, respectively (Fig. 2).

204 As truncated StkA and StkB may retain part of their functions, these insertions could be leaky or even

205 gain-of-function mutations. In addition, $s t k A, s t k B$ and $s t k C$ may form an operon (Fig. 2) and both

206 insertions could be polar on downstream genes. The previous stkA mutant harbors a $\mathrm{Tn}$ insertion as

207 well (Dana \& Shimkets 1993b). To clarify the roles of the stk genes in EPS production, in-frame

208 deletions of these genes were constructed (See Material and Methods). YZ812, YZ813 and YZ910

209 deleted $s t k A, s t k B$ and $s t k C$, respectively (Table 1). EPS production by these strains was examined by

$210 \mathrm{CW}$ binding (Fig. 1C). $\Delta s t k A$ exhibited more whereas $\Delta s t k B$ and $\Delta s t k C$ exhibited less fluorescence than

211 the WT in this assay. In addition, EPS levels of the stk deletion mutants were quantified by a liquid dye

212 binding assay (Black \& Yang 2004). As show in Fig. 3, the $\triangle s t k A$ mutant increased EPS production

213 over the wild type by about $50 \%$. The $\triangle s t k B$ and $\Delta s t k C$ mutants produced about $50 \%$ and $25 \%$ less than

214 the WT, respectively. The results in Figs. 1C and 3 with the in-frame deletion mutants clearly implicate

215 stk genes in M. xanthus EPS production. StkA is likely a negative regulator as its absence results in

216 EPS overproduction. The roles of StkB and StkC are less clear as the deletion of their genes led to

217 intermediate EPS phenotypes. Phenotypic comparisons also indicate that the $s t k B$ insertion in BY129 
218 and BY1129 is likely polar because it led to a more severe EPS defect than the deletion of either stkB

219 or stkC. There are two additional ORFs (MXAN_3471 and MXAN_3472) upstream of and in the same

220 orientation as StkA (Goldman et al. 2006). Their in-frame deletions resulted in no alteration in $M$.

221 xanthus EPS production or any other phenotype examined (results not shown) and these two genes are

222 not discussed in this manuscript.

\section{StkA functions downstream of PilA but upstream of Dif Proteins}

224 EPS production is regulated in part by the Dif pathway (Black et al. 2006; Yang et al. 2014). T4P are

225 proposed to perceive and relay signals downstream to Dif proteins to promote EPS production (Black

226 et al. 2006). The relationship of StkA with Dif was examined by the construction of a $\Delta$ difA $\Delta s t k A$

227 mutant. In addition, a $\triangle p i l A \Delta s t k A$ mutant was constructed to confirm the suppression of $\triangle p i l A$ by the

$228 s t k A$ deletion. As shown in Fig. 4, the $\triangle p i l A \Delta s t k A$ double mutant (YZ901) produced more EPS

229 similarly as the $\Delta s t k A$ single mutant (YZ812), indicating the suppression of $\triangle p i l A$ by stkA null

230 mutations. On the other hand, the $\triangle$ difA $\triangle s t k A$ double mutant (YZ932) appeared similar to the $\Delta$ difA

231 single mutant (YZ601) with both producing very little EPS (Fig. 4). The finding that the $4 s t k A$

232 mutation is epistatic to a $\triangle p i l A$ but not a $\triangle$ difA mutation led to the conclusion that StkA functions

233 between T4P and the Dif pathway in the regulation of EPS production in M. xanthus.

\section{4 stk mutants show defects in motility}

235 The surface motility of the $s t k$ deletions were examined first on regular agar plates $(1.5 \%$ agar $)$ which

236 allow both A and S motility to contribute (Shi \& Zusman 1993) (Fig. 5A). Compared to the WT, the

237 colony of the $\Delta s t k C$ mutant (YZ910) appears only slightly smaller, consistent with the slight effect of

238 the $s t k C$ deletion on EPS levels (Figs $1 \mathrm{C}$ and 3). The colony morphology of the $\triangle s t k C$ mutant was also 
239 highly similar to that of WT in its yellow pigmentation, high opacity as well as its rough surface and

240 jagged edges. $\triangle s t k A$ and especially $\triangle s t k B$ mutants showed a more diminished ability to spread on hard

241 agar surfaces as their swarming colonies appeared smaller than that of the WT. The surface of the

$242 \Delta s t k A$ mutant colony is rougher and more elevated than that of the WT. The colony of $\Delta s t k B$ is

243 smoother, glossier and flatter than the WT. These observations are consistent with the $\Delta s t k A$ mutant

244 overproducing and the $\triangle s t k B$ mutants significantly underproducing EPS in comparison with the WT.

245 Because EPS is essential for the T4P-mediated S motility, plates with $0.4 \%$ agar (soft agar

246 plates) were used to examine S motility more specifically (Shi \& Zusman 1993) (Fig. 5B). The size of

247 the swarming colony of the $\Delta s t k C$ mutant is similar to that of the WT, indicating no obvious S-motility

248 defect. The spreading of $\Delta s t k B$ and $\Delta s t k A$ mutants, especially the latter, was defective in comparison

249 with the WT. Interestingly, except the size, the colony morphology of the $\Delta s t k A$ mutant more closely

250 resembled that of the WT. There were obvious swarming zones or rings for strains of the WT and the

$251 \Delta s t k A$, but not for those of the $\Delta s t k B$ and the $\Delta s t k C$. Overall, these results indicate that StkA and StkB

252 are more important players in S-motility whereas StkC may influence the organization of swarms but

253 not the overall rate of swarming by S motility.

254 stk mutants show defects in developmental aggregation consistent with their EPS

255 phenotypes

$256 s t k$ mutants were examined for development under nutrient limitation (Fig. 5C). With respect to both

257 fruiting body morphology and the completeness of aggregation, the $\Delta s t k A$ mutant was the most

258 defective followed by $\Delta s t k B$ and $\Delta s t k C$. While some of the aggregates of $\Delta s t k C$ are elongated, their

259 distribution and number are the most similar to those of the WT. The aggregates formed by the $\Delta s t k B$

260 mutant darkened as those of the WT, but they appeared less well organized and more variable in size 
261 and number. While the $\Delta s t k A$ mutant showed signs of aggregation in the center of the bacterial lawn,

262 these aggregates are smaller and more numerous. On the edge of the lawn, $\Delta s t k A$ cells appeared to

263 move outward with no signs of aggregation. These results are consistent with the varying degrees of

264 EPS defects of stk mutants as $\Delta s t k A$ had the most severe EPS phenotype, followed by $\Delta s t k B$ and $\Delta s t k C$. 


\section{DISCUSSION \& CONCLUSIONS}

267 To summarize, two stk insertion mutants were isolated in two separate genetic screens based on their

268 altered EPS phenotypes. Further analysis indicated that both a stkA in-frame deletion and an insertion

269 resulted in EPS overproduction in the WT background. While stkA mutations suppressed ApilA in EPS

270 regulation, they failed to restore EPS production to a $\triangle d i f A$ mutant. Both $s t k B$ and $s t k C$ deletions

271 resulted in varying reductions in EPS production and surface motility, consistent with the correlation

272 between EPS and motility observed previously (Xu et al. 2005). These results established that the three

273 genes at the stk locus are important for EPS production in M. xanthus albeit to different degrees. The

274 observation that the $s t k A$ mutant displayed reduced swarming by S motility (Fig. 5B) indicates that

275 optimal S motility requires a fine balance or a tight regulation of EPS production. Too little or too

276 much apparently results in reduced efficiency of spreading through S motility (Xu et al. 2005).

StkA is a member of the Hsp70 protein family (Weimer et al. 1998). The prototype Hsp70 is

278 the E. coli chaperone DnaK (Genevaux et al. 2007). It functions as part of a molecular machine with

279 DnaJ and GrpE, its partners or co-chaperones, to facilitate the folding of nascent polypeptides and the

280 refolding of denatured or misfolded proteins. These proteins are induced by heat shock and they confer

281 thermotolerance to E. coli once induced. Multiple lines of evidence indicate that StkA is a negative

282 regulator of EPS production in M. xanthus. Previously, StkA was not found to be induced by heat

283 shock (Otani et al. 2001), thus not a typical bacterial Hsp70. Instead, our genetic epistasis results here

284 support a model wherein StkA functions as a negative regulator downstream of T4P but upstream of

285 the Dif chemotaxis protein in the EPS regulatory pathway. Previous results left little doubt that StkA is

286 critical for the production of fibrils (Dana \& Shimkets 1993b), of which EPS is a major constituent 
287 (Behmlander \& Dworkin 1994). The results here demonstrate that StkA itself is a negative regulator of 288 EPS and it lies downstream of T4P and upstream of Dif in the EPS regulatory pathway in M. xanthus. As a component of the EPS signaling pathway, StkA may modulate the function of other EPS regulators in a chaperone-like capacity or it may act directly as a signaling protein in an unknown 291 manner. In this context, it is noted that SglK, another M. xanthus Hsp70 homologue, has the opposite 292 function in EPS regulation when compared with StkA (Weimer et al. 1998; Yang et al. 1998a). That is, 293 a sglK mutant is EPS- and has no S motility. It is surprising that there are 15 Hsp70-like proteins 294 encoded by the M. xanthus genome (Goldman et al. 2006). This is in contrast to E. coli which codes 295 three Hsp70 members on its genome (Genevaux et al. 2007). Besides the canonical heat shock protein DnaK, HscA and $\mathrm{HscC}$ are the other two Hsp70-like proteins in this enteric bacterium. HscA is a specialized chaperone that facilitates the assembly and maturation of iron-sufur [Fe-S] proteins. HscC appears to be involved in response to more general stress including UV exposure through mechanisms that is not entirely clear. If StkA functions as a chaperone like HscA, it may facilitate a negative regulator of EPS to attain or maturate to its native and active state. If StkA is a signaling protein, it may function in a similar fashion as Ssz1, a regulatory Hsp70 in yeast (Prunuske et al. 2012). The identification of the direct target of StkA will provide insights into the mechanisms of EPS regulation by this member of Hsp70 proteins in M. xanthus.

It is unclear whether StkB and StkC function in a regulatory or a biosynthetic capacity. In comparison with the $s t k B$ and the $s t k C$ deletions, the $s t k B 1:: T n$ mutant has a more severe EPS phenotype (Fig. 1). This suggests that the $s t k B 1:: T n$ mutation is polar on $s t k C$ (Fig. 2) and that StkB and StkC have overlapping or redundant functions. The homology of StkC to PhaE (Han et al. 2010) may be taken as circumstantial evident that StkC as well as StkB are EPS biosynthetic enzymes.

09 However, the homology of StkB to NSLTPs (Lev 2010; Schroeder et al. 2007) leads to ambiguities if 
310 there is indeed an overlapping function for these two proteins. NSLTPs are involved not only in lipid

311 metabolism but also in cell signaling (Lev 2010; Schroeder et al. 2007), which could mean both

312 function in cell signaling instead of the biosynthetic process. Further investigations are necessary to

313 better understand the roles of StkB and StkC in M. xanthus EPS production. 
314 Acknowledgements. This work was partially supported by the National Science Foundation Grant

315 MCB-1417726, the National Institute of Health Grant GM071601 and Fralin Life Science Institute to

316 ZY. RAW was partially supported by the Post Baccalaureate Research and Education Program (PREP)

317 and the Multicultural Academic Opportunities Program (MAOP) at Virginia Tech. The funders had no

318 role in study design, data collection and analysis, decision to publish, or preparation of the manuscript. 
Behmlander RM, and Dworkin M. 1994. Biochemical and structural analyses of the extracellular matrix fibrils of Myxococcus xanthus. J Bacteriol 176:6295-6303.

Bellenger K, Ma X, Shi W, and Yang Z. 2002. A CheW homologue is required for Myxococcus xanthus fruiting body development, social gliding motility, and fibril biogenesis. J Bacteriol 184:5654-5660.

Black WP, Schubot FD, Li Z, and Yang Z. 2010. Phosphorylation and dephosphorylation among Dif chemosensory proteins essential for exopolysaccharide regulation in Myxococcus xanthus. $J$ Bacteriol 192:4267-4274.

Black WP, Xu Q, Cadieux CL, Suh S-J, Shi W, and Yang Z. 2009. Isolation and characterization of a suppressor mutation that restores Myxococcus xanthus exopolysaccharide production. Microbiol 155:3599-3610.

Black WP, Xu Q, and Yang Z. 2006. Type IV pili function upstream of the Dif chemotaxis pathway in Myxococcus xanthus EPS regulation. Mol Microbiol 61:447-456.

Black WP, and Yang Z. 2004. Myxococcus xanthus chemotaxis homologs DifD and DifG negatively regulate fibril polysaccharide production. J Bacteriol 186:1001-1008.

Campos JM, and Zusman DR. 1975. Regulation of development in Myxococcus xanthus: effect of 3':5'cyclic AMP, ADP, and nutrition. Proc Natl Acad Sci USA 72:518-522.

Dana JR, and Shimkets LJ. 1993a. Regulation of cohesion-dependent cell interactions in Myxococcus xanthus. J Bacteriol 175:3636-3647.

Dana JR, and Shimkets LJ. 1993b. Regulation of cohesion-dependent cell interactions in Myxococcus xanthus. J Bacteriol 175:3636-3647.

Genevaux P, Georgopoulos C, and Kelley WL. 2007. The Hsp70 chaperone machines of Escherichia coli: a paradigm for the repartition of chaperone functions. Mol Microbiol 66:840-857.

Goldman BS, Nierman WC, Kaiser D, Slater SC, Durkin AS, Eisen JA, Ronning CM, Barbazuk WB, Blanchard M, Field C, Halling C, Hinkle G, Iartchuk O, Kim HS, Mackenzie C, Madupu R, Miller N, Shvartsbeyn A, Sullivan SA, Vaudin M, Wiegand R, and Kaplan HB. 2006. Evolution of sensory complexity recorded in a myxobacterial genome. Proc Natl Acad Sci USA 103:15200-15205.

Hagen DC, Bretscher AP, and Kaiser D. 1978. Synergism between morphogenetic mutants of Myxococcus xanthus. Dev Biol 64:284-296.

Han J, Hou J, Liu H, Cai S, Feng B, Zhou J, and Xiang H. 2010. Wide distribution among halophilic archaea of a novel polyhydroxyalkanoate synthase subtype with homology to bacterial type III synthases. Appl Environ Microbiol 76:7811-7819.

Julien B, Kaiser AD, and Garza A. 2000. Spatial control of cell differentiation in Myxococcus xanthus. Proc Natl Acad Sci USA 97:9098-9103.

Kaiser D. 1979. Social gliding is correlated with the presence of pili in Myxococcus xanthus. Proc Natl Acad Sci USA 76:5952-5956.

Kim SH, Ramaswamy S, and Downard J. 1999. Regulated exopolysaccharide production in Myxococcus xanthus. J Bacteriol 181:1496-1507.

Lev S. 2010. Non-vesicular lipid transport by lipid-transfer proteins and beyond. Nat Rev Mol Cell Biol 11:739-750.

Li Y, Sun H, Ma X, Lu A, Lux R, Zusman D, and Shi W. 2003. Extracellular polysaccharides mediate pilus retraction during social motility of Myxococcus xanthus. Proc Natl Acad Sci USA 100:5443-5448. 
Lu A, Cho K, Black WP, Duan XY, Lux R, Yang Z, Kaplan HB, Zusman DR, and Shi W. 2005. Exopolysaccharide biosynthesis genes required for social motility in Myxococcus xanthus. Mol Microbiol 55:206-220.

Mauriello EM, Mignot T, Yang Z, and Zusman DR. 2010. Gliding motility revisited: how do the myxobacteria move without flagella? Microbiol Mol Biol Rev 74:229-249.

Otani M, Tabata J, Ueki T, Sano K, and Inouye S. 2001. Heat-shock-induced proteins from Myxococcus xanthus. J Bacteriol 183:6282-6287.

Prunuske AJ, Waltner JK, Kuhn P, Gu B, and Craig EA. 2012. Role for the molecular chaperones Zuo1 and Ssz1 in quorum sensing via activation of the transcription factor Pdr1. Proc Natl Acad Sci USA 109:472-477.

Rubin EJ, Akerley BJ, Novik VN, Lampe DJ, Husson RN, and Mekalanos JJ. 1999. In vivo transposition of mariner-based elements in enteric bacteria and mycobacteria. Proc Natl Acad Sci USA 96:1645-1650.

Sambrook J, and Russell DW. 2001. Molecular cloning: a laboratory manual $3^{\text {rd }}$ edition. Cold Spring Harbor, NY: Cold Spring Harbor.

Schroeder F, Atshaves BP, McIntosh AL, Gallegos AM, Storey SM, Parr RD, Jefferson JR, Ball JM, and Kier AB. 2007. Sterol carrier protein-2: new roles in regulating lipid rafts and signaling. Biochim Biophys Acta 1771:700-718.

Shi W, and Zusman DR. 1993. The two motility systems of Myxococcus xanthus show different selective advantages on various surfaces. Proc Natl Acad Sci USA 90:3378-3382.

Ueki T, Inouye S, and Inouye M. 1996. Positive-negative KG cassettes for construction of multi-gene deletions using a single drug marker. Gene 183:153-157.

Vlamakis HC, Kirby JR, and Zusman DR. 2004. The Che4 pathway of Myxococcus xanthus regulates type IV pilus-mediated motility. Mol Microbiol 52:1799-1811.

Wall D, and Kaiser D. 1998. Alignment enhances the cell-to-cell transfer of pilus phenotype. Proc Natl Acad Sci USA 95:3054-3058.

Wallace RA, Black WP, Yang X, and Yang Z. 2014. A CRISPR with Roles in Myxococcus xanthus Development and Exopolysaccharide Production. J Bacteriol 196:4036-4043.

Weimer RM, Creighton C, Stassinopoulos A, Youderian P, and Hartzell PL. 1998. A chaperone in the HSP70 family controls production of extracellular fibrils in Myxococcus xanthus. J Bacteriol 180:5357-5368.

Xu Q, Black WP, Ward SM, and Yang Z. 2005. Nitrate-dependent activation of the Dif signaling pathway of Myxococcus xanthus mediated by a NarX-DifA interspecies chimera. J Bacteriol 187:6410-6418.

Yang Z, Geng Y, and Shi W. 1998a. A DnaK homolog in Myxococcus xanthus is involved in social motility and fruiting body formation. J Bacteriol 180:218-224.

Yang Z, Geng Y, Xu D, Kaplan HB, and Shi W. 1998b. A new set of chemotaxis homologues is essential for Myxococcus xanthus social motility. Mol Microbiol 30:1123-1130.

Yang Z, and Higgs PI. 2014. Myxobacteria. Norfolk, UK: Caister Academic Press. p 235.

Yang Z, Li CY, Friedrich C, and Sogaard-Andersen L. 2014. Type IV pili and exopolysaccharidedependent motility in Myxococcus xanthus. In: Yang Z, and Higgs PI, eds.: Caister Academic Press, 183-198.

Yang Z, and Li Z. 2005. Demonstration of interactions among Myxococcus xanthus Dif chemotaxislike proteins by the yeast two-hybrid system. Arch Microbiol 183:243-252.

Yang Z, Ma X, Tong L, Kaplan HB, Shimkets LJ, and Shi W. 2000. Myxococcus xanthus dif genes are required for biogenesis of cell surface fibrils essential for social gliding motility. $J$ Bacteriol 182:5793-5798. 
411 Youderian P, Burke N, White DJ, and Hartzell PL. 2003. Identification of genes required for 412 adventurous gliding motility in Myxococcus xanthus with the transposable element mariner. $413 \quad$ Mol Microbiol 49:555-570.

414 Zusman DR, Scott AE, Yang Z, and Kirby JR. 2007. Chemosensory pathways, motility and 415 development in Myxococcus xanthus. Nat Rev Microbiol 5:862-872.

416 
Table $\mathbf{1}$ (on next page)

Strains and plasmids

Table 1 : Plasmids and $M$. xanthus strains used in this study 
Table 1: Plasmids and $M$. xanthus strains used in this study

\begin{tabular}{|c|c|c|}
\hline Plasmid & Genotype or description & Source \\
\hline pBJ113 & M. xanthus gene replacement vector & $\begin{array}{l}\text { (Julien et al. } \\
2000) \\
\text { (Julien et al. }\end{array}$ \\
\hline pBJ114 & M. xanthus gene replacement vector & 2000) \\
\hline pLZ407 & stk $A$ in-frame deletion $(\Delta s t k A)$ in $\mathrm{pBJ} 113$ & This study \\
\hline pLZ429 & stkB in-frame deletion $(\Delta s t k B)$ in $\mathrm{pBJ} 114$ & This study \\
\hline pAM108 & stk $C$ in-frame deletion $(\Delta s t k C)$ in pBJ113 & $\begin{array}{l}\text { This study } \\
\text { (Rubin et al. }\end{array}$ \\
\hline pMycoMar & magellan4 mutagenesis vector & 1999) \\
\hline \multicolumn{3}{|c|}{ M. xanthus strain } \\
\hline BY129 & stkB1::Tn in YZ101 & this study \\
\hline BY801 & $\triangle p i l A$ stkA2::Tn & this study \\
\hline BY1129 & stkB1::Tn & this study \\
\hline BY1801 & stkA2::Tn & $\begin{array}{l}\text { this study } \\
\text { (Kaiser }\end{array}$ \\
\hline DK1622 & Wild type (WT) & $\begin{array}{l}\text { 1979) } \\
\text { (Wall \& } \\
\text { Kaiser }\end{array}$ \\
\hline DK10407 & $\Delta p i l A:: T e t$ & $\begin{array}{l}\text { 1998) } \\
\text { (Black et }\end{array}$ \\
\hline YZ101 & $\triangle$ difA cheW7-1 ( $\triangle$ difA suppressor strain) & $\begin{array}{l}\text { al. 2009) } \\
\text { (Xu et al. }\end{array}$ \\
\hline YZ601 & $\Delta$ difA & $\begin{array}{l}2005) \\
\text { (Black \& } \\
\text { Yang }\end{array}$ \\
\hline YZ603 & $\Delta$ difE & 2004) \\
\hline YZ690 & $\Delta p i l A$ & this study \\
\hline YZ812 & $\Delta s t k A$ & this study \\
\hline YZ813 & $\Delta s t k B$ & this study \\
\hline YZ901 & $\Delta$ pilA $\Delta s t k A$ & this study \\
\hline YZ910 & $\Delta s t k C$ & this study \\
\hline YZ932 & $\triangle \operatorname{difA} \Delta s t k A$ & this study \\
\hline
\end{tabular}




\section{1}

EPS production of stk mutants.

Figure 1. EPS production of stk mutants.

Five microliter aliquots of cell suspension at $5 \times 10^{9} \mathrm{cells} / \mathrm{ml}$ of an indicated strain were spotted onto CYE plates with the fluorescent dye calcofluor white (CW) and florescence was documented under UV illumination after 6 days of incubation at $32^{\circ} \mathrm{C}$ (See Materials \& Methods). A. stkA insertion suppressed pilA deletion. B. stkB insertion results in EPS defect. C. Deletions of stk genes affect EPS levels to different extent. Strains: DK1622 (wild type),

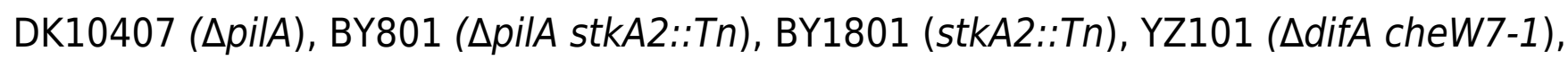

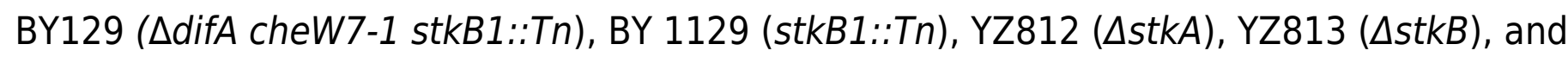
YZ910 ( $\Delta s t k C)$.
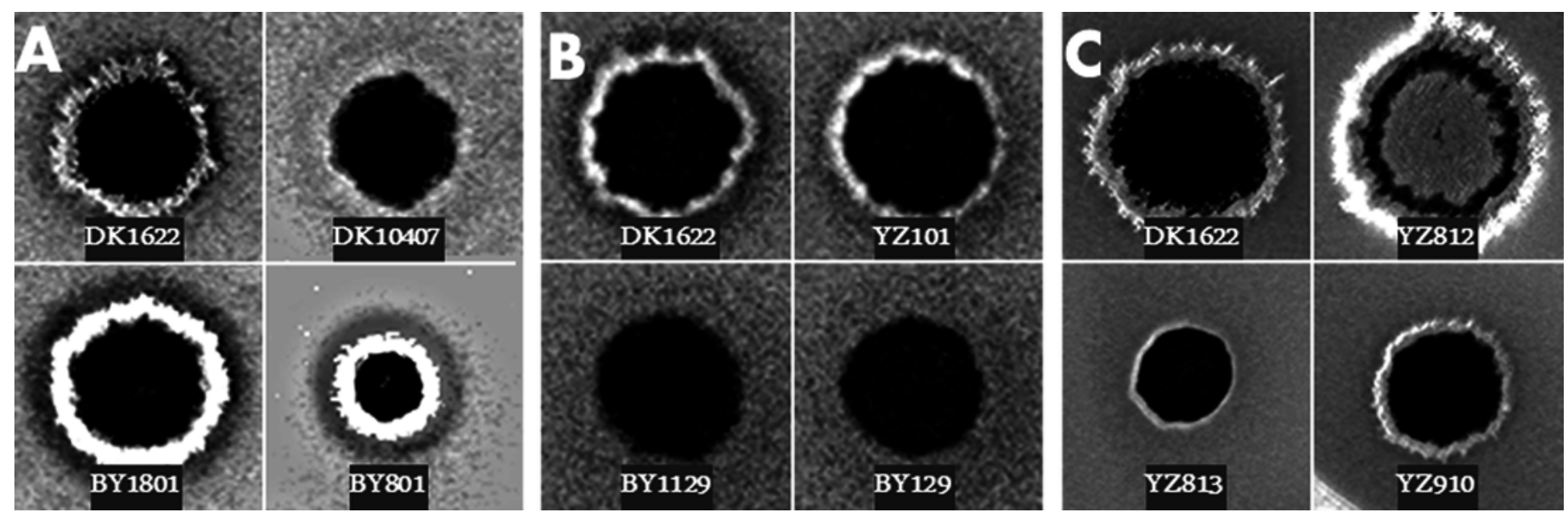
2

. M. xanthus stk locus X

Figure 2. M. xanthus stk locus.

The stk region shown is $2.54 \mathrm{~kb}$ with the ORFs of StkA, StkB and StkC indicated by open arrows approximately to scale. The inverted arrows indicate positions of $\mathrm{Tn}$ insertions in stkA and stkB in BY801 and BY129, respectively.

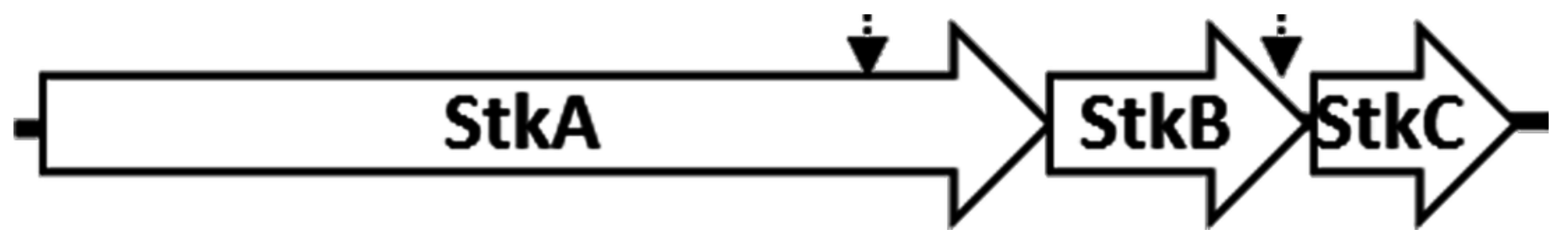




\section{3}

The stkA mutant produced more EPS whereas -and the stkB and stkC mutants produced less

Figure 3. The stkA mutant produced more EPS whereas -and the stkB and stkC mutants produced less.

EPS levels were quantified by a trypan blue binding assay (See Materials \& Methods). The amount of EPS for each strain was compared to that of the WT (DK1622) which was normalized to a value of one. Other strains are YZ812 $(\Delta s t k A), Y Z 813(\Delta s t k B)$, and YZ910 $(\Delta s t k C)$ with the EPS strain YZ603 $(\Delta d i f E)$ as a control.

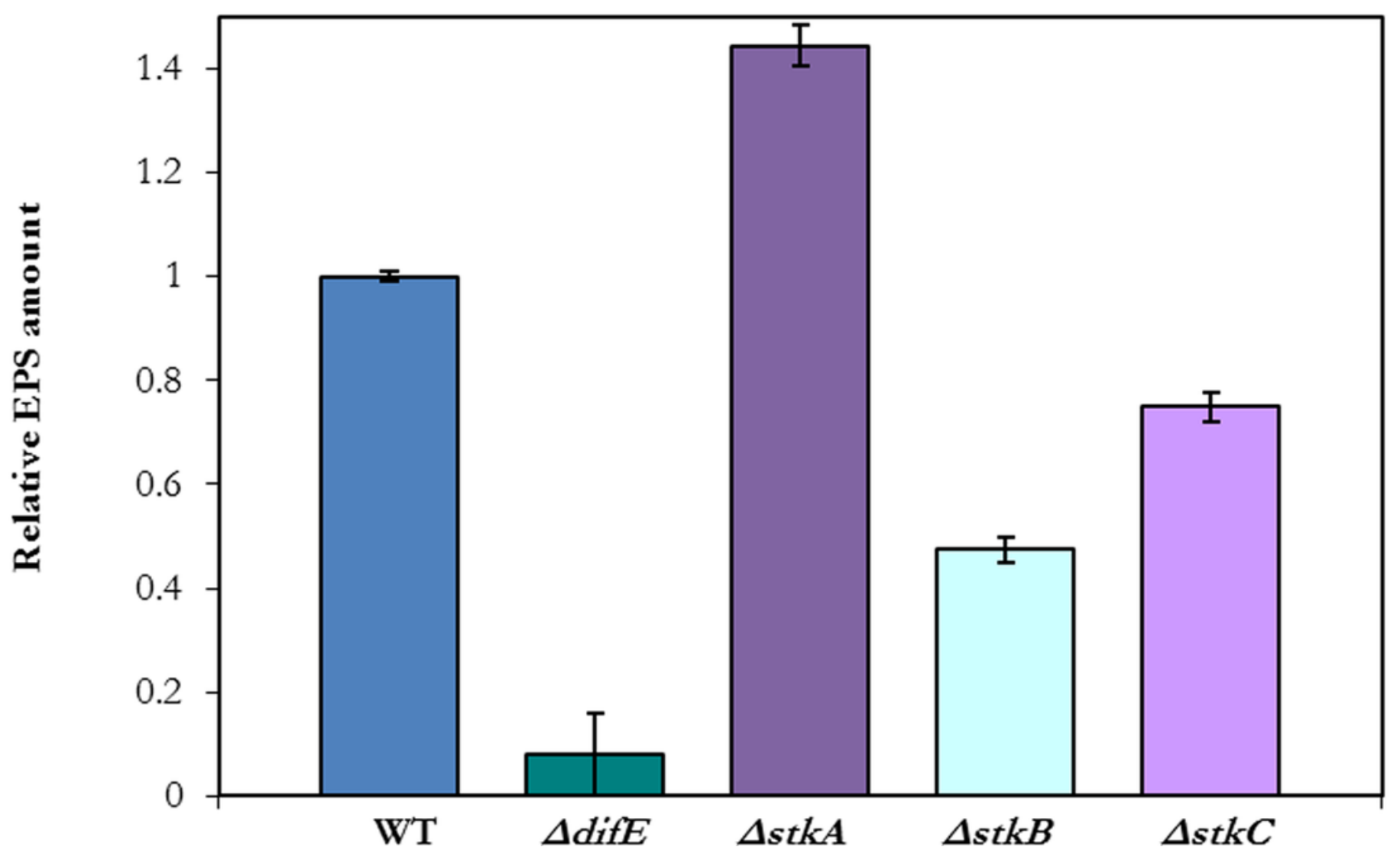


4

$\Delta$ stkA suppresses $\Delta$ pilA but not $\Delta$ difA

Figure 4. $\Delta$ stkA suppresses $\Delta$ pilA but not $\Delta$ difA.

EPS levels were quantified by a trypan blue binding assay as in Fig. 4. The strains are

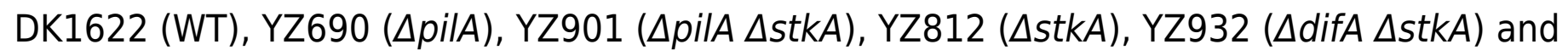
YZ601 ( $\triangle$ difA).

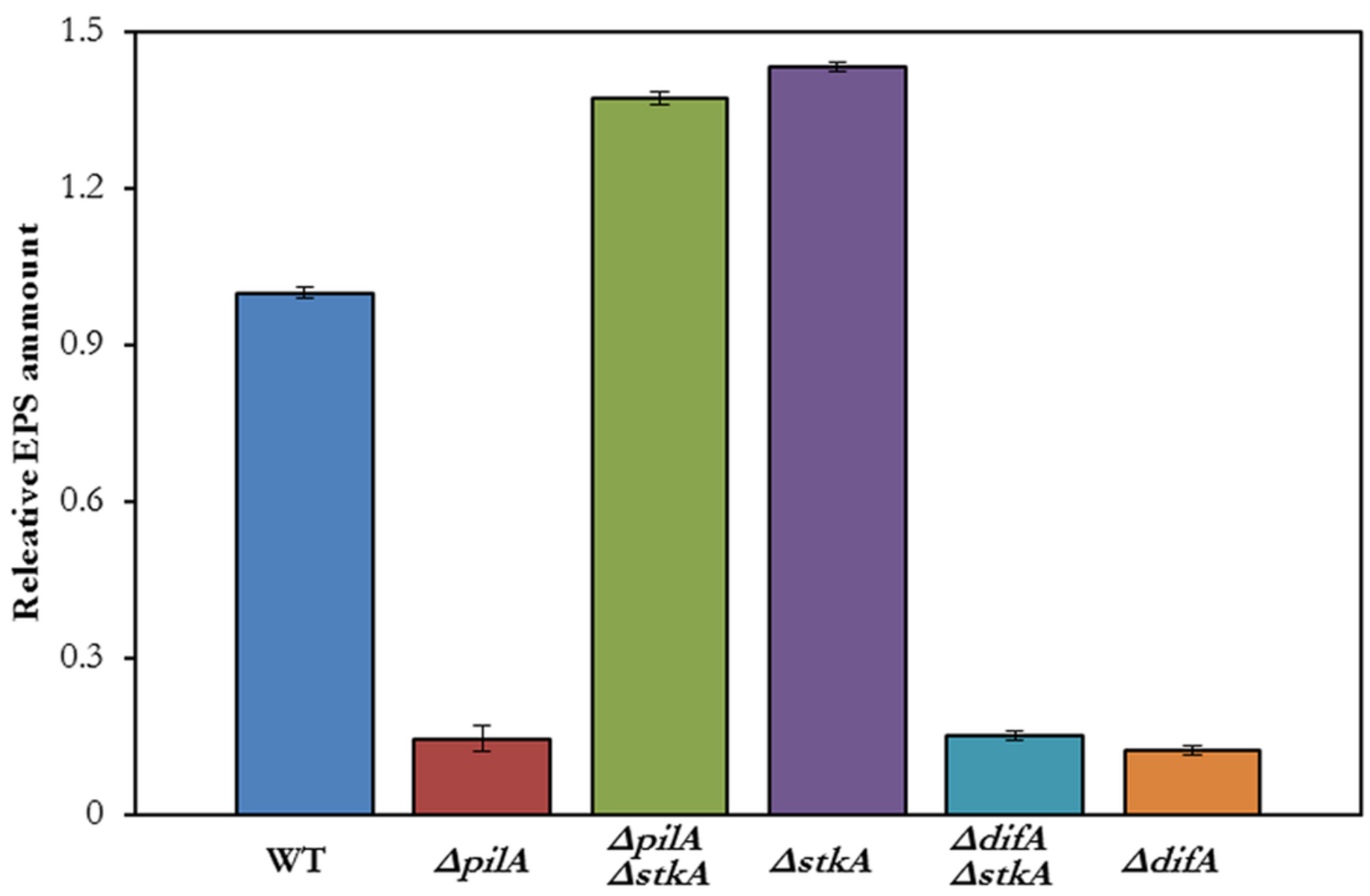




\section{5}

Motility and developmental aggregation of stk mutants

Figure 5. Motility and developmental aggregation of stk mutants. A $5 \mu$ aliquot of the cell suspension at $5 \times 10^{9}$ cells $/ \mathrm{ml}$ for each strain were plated in the center of a CYE plate with $1.5 \%(\mathbf{A})$ or $0.4 \%$ agar $(\mathbf{B})$ to examine motility. The same amount of cells of a strain was spotted onto a CF plate to examine development (C). Results were documented after incubation at $32{ }^{\circ} \mathrm{C}$ (See Materials and Methods). The scale bars in all three panels represent $1 \mathrm{~cm}$. Indicated on the top of the figure are the strains used for all three panels: DK1622

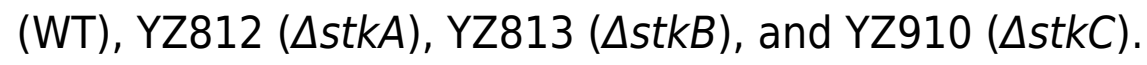
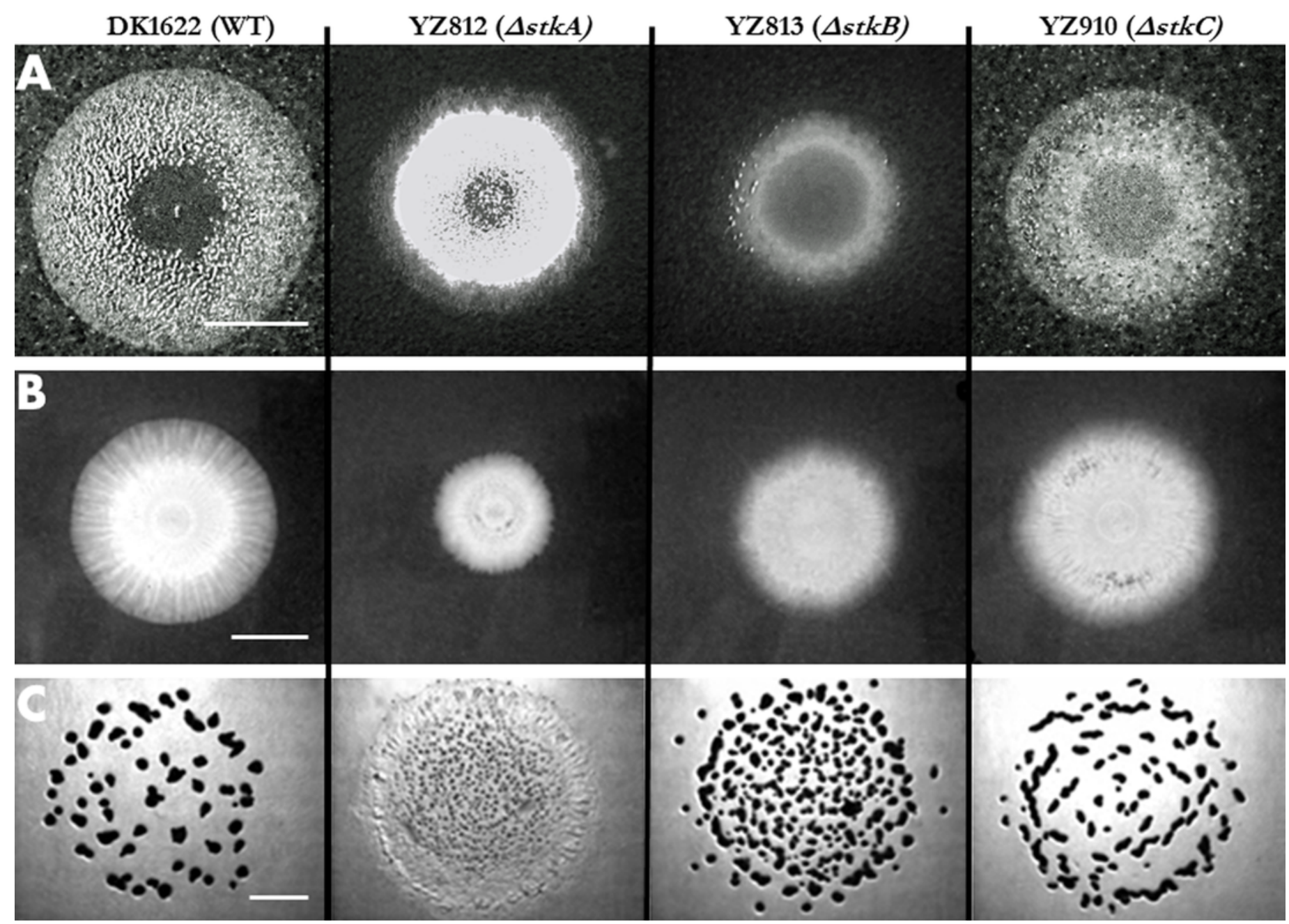